\title{
Combined oral contraceptives, smoking, and cardiovascular risk
}

\author{
Timothy M M Farley, Olav Meirik, C Lan Chang, Neil R Poulter
}

\begin{abstract}
Study objective-To assess age specific incidence and mortality of stroke, acute myocardial infarction (AMI), and idiopathic venous thromboembolism (VTE) associated with use of modern low dose combined oral contraceptives (OCs) and the interaction with smoking.
\end{abstract}

Design-Hospital-based case-control study.

Setting-Hospitals in Oxford region in the United Kingdom, which covered a defined population, during the period 1989-1993.

Methods-Relative risk estimates from the WHO Collaborative Study and observed incidence rates from the Oxford region were used to estimate age specific incidence of each disease among women without cardiovascular risk factors and model total cardiovascular incidence and mortality.

Results-Among women who did not use OCs, smoke nor had any other cardiovascular risk factors, total incidence of stroke and AMI were less than 2 events per 100000 woman years in those aged 20-24 years and rose exponentially with age to 8 events per 100000 among women aged 40-44 years. Incidence of idiopathic VTE among women who did not use OCs rose linearly with age (from 3.3 per 100000 at ages 20-24 years to 5.8 per 100000 at ages 40-44 years). The increased risk of idiopathic VTE associated with OC use among non-smokers constituted over $90 \%$ of all cardiovascular events for women aged 20-24 years and more than $60 \%$ in those aged 40-44 years. Fatal cardiovascular events were dominated by haemorrhagic stroke and AMI, and among OC users who smoked these two diseases accounted for $80 \%$ of cardiovascular deaths among women aged 20-24 years, rising to $97 \%$ among those aged $40-44$ years. Cardiovascular mortality associated with smoking was greater than that associated with OC use at all ages. Attributable risk associated with OC use was 1 death per 370000 users annually among women aged 20-24 years, 1 per 170000 at ages 30-34 years, and 1 per 37000 at ages 40-44 years. Among smokers, the cardiovascular mortality attributable to OC use was estimated to be about 1 per 100000 users annually among women aged less than 35 years, and about 1 per 10000 users annually among those above the age of 35 years.

Conclusion-The incidence of fatal cardiovascular events among women aged less than 35 years is low. The VTE risk associated with OC use is the largest contributor to $O C$ induced adverse effects. The potentially avoidable excess VTE risk associated with the newer progestogens desogestrel and gestodene would account for a substantial proportion of total cardiovascular morbidity in this age group. For women over age 35 years the absolute risks associated with $O C$ use and smoking are greater because of the steeply rising incidence of arterial diseases. The combination of smoking and OC use among such women is associated with particularly increased risks. Any potential reduction in AMI or stroke risk with use of third generation OCs would be a more important consideration among older compared with younger women, particularly if they smoke. However, the mortality associated with smoking is far greater than that associated with OC use (of any type) at all ages.

(F Epidemiol Community Health 1998;52:775-785)

The WHO Collaborative Study of Cardiovascular Disease and Steroid Hormone Contraception has recently provided information on the risks of idiopathic venous thromboembolism (VTE), ${ }^{12}$ ischaemic and haemorrhagic stroke (IS and HS), ${ }^{34}$ and acute myocardial infarction $(\mathrm{AMI})^{5}$ associated with use of combined oral contraceptives (OCs) in selected developing and European countries. This paper estimates the age specific incidence and mortality of first-time events of all four diseases among women with no cardiovascular risk factors and models the risks attributable to OC use, smoking and the combination of the two. The model is based on relative risk estimates from the WHO Study and observed disease incidence from the Oxford region in the United Kingdom. It provides a framework to assess OC associated cardiovascular risks in populations with different incidences and patterns of risk factors. Similarly, the impact of effective screening of OC users for predisposing factors on the incidence of non-fatal and fatal cardiovascular events, and the impact of potential differences between OC types on the risks of VTE, stroke, and AMI are assessed.

\section{Methods}

GENERAL DESIGN

The WHO Study was a hospital-based casecontrol study of VTE, IS, HS, and AMI events among women of reproductive age conducted in 21 centres in 17 countries between 1989 and 1993. Case accrual continued up to 1995 in 
some centres for AMI. Cases were women aged 20-44 years admitted to hospital with a first time diagnosis of one of the study diseases. For each case up to three controls matched by age, hospital, and date of admission were identified and interviewed while in hospital using identical procedures. Non-hospitalised or rapidly fatal cases (those who died within 24 hours of hospital admission) were not included, and proxy respondents were used to provide information on those cases unable to complete the study questionnaire. ${ }^{6}$ Cases and controls with a history of hysterectomy, menopause or any of the study diseases, or who had been pregnant in the previous six weeks, or had a recent history (within six weeks) of surgery, trauma, or prolonged bed rest were not eligible. Full details of the study procedures and methods have been published elsewhere. ${ }^{7}$

ESTIMATED INCIDENCE RATES

Cases in the Oxford region of the UK were identified from all hospitals that admitted acute cases and covered a defined geographical area (former Oxford Regional Health Authority) with known age and sex disaggregated population $\operatorname{size}^{8}$ (average mid-year population size over the period of case accrual included 494000 women aged $20-44$ years). It was the only centre that participated in the study for which the study base population could be defined and thus provide estimates of disease incidence rates. To estimate the age specific VTE incidence among non-OC users we used the overall age specific VTE incidence, risk estimates according to OC type, ${ }^{2}$ and age specific prevalence and type of OC use among all 649 hospital controls matched to cases in the Oxford region. In each age stratum, the total $\left(I_{\text {tot }}\right)$ incidence rate and incidence in non-OC users $\left(I_{0}\right)$ are related by

$$
I_{\text {tot }}=p_{0} I_{0}+p_{1} I_{1}+p_{2} I_{2}
$$

where $p_{1}$ and $p_{2}$ are the prevalence of use of the two OC types, $I_{1}$ and $I_{2}$ the corresponding VTE incidence rates in such users, and $p_{0}=1-p_{1}-p_{2}$ the proportion of non-OC users. The VTE incidence in users of each OC type is $I_{r}=R_{r} I_{0}$ where $R_{r}$ is risk relative to non-users, $r=1,2$. Thus $I_{0}=I_{\text {tor }} /\left(p_{0}+p_{1} R_{1}+p_{2} R_{2}\right)$ in each age stratum. The age specific incidence rates among non-OC users $\left(I_{0}\right)$ were smoothed by fitting a linear trend.

A similar procedure was followed to estimate age specific incidence of IS, HS, and AMI among non-users without cardiovascular risk factors, but considering patterns of smoking and reported prevalence of other major risk factors for arterial disease (hypertension, diabetes mellitus, hyperlipidaemia) in addition to OC use. As the majority of OC users in the UK used low oestrogen dose preparations, and there was little observed difference in risk for these three diseases according to progestogen type, the same risk ratios were assumed for all OC users. Based on the results from the WHO Study, ${ }^{3-5}$ risks among OC users who smoked were assumed to be the product of risks associated with smoking and OC use separately. Estimated age specific incidence rates among women who did not use OCs nor smoke were smoothed by fitting a weighted exponential trend with weights inversely proportional to variance. As the study was hospital-based and excluded cases who were not hospitalised or died within 24 hours of admission, incidence rates were scaled up by a factor corresponding to the estimated proportion of cases not included. This factor was estimated from the mortality rates and proportion of fatal cases who died in the first 24 hours in the MONICA study. $^{9} 10$

MODELLING OC AND SMOKING ASSOCIATED INCIDENCE OF TOTAL (NON-FATAL AND FATAL) CARDIOVASCULAR EVENTS

Stroke and AMI risks among OC users and/or smokers were based on estimated risks for low oestrogen dose OCs, the most commonly used type of OC worldwide. The VTE risk was based on that for low dose levonorgestrel containing OCs. ${ }^{2}$ Total age specific incidence of non-fatal and fatal cardiovascular events were computed using HS, IS, AMI, and VTE incidence among non-OC users who did not smoke, the relative risk estimates for smokers and OC users, multiplicative risks for OC users who smoked, and disease specific 28 day case fatality rates $\left(50 \%,{ }^{9} 30 \%,{ }^{9} 46 \%{ }^{10}\right.$ and $2 \%,{ }^{11}$ respectively). Age standardised incidence rates were based on the age distribution of women aged 20-44 years, ${ }^{8}$ or the estimated age distribution of OC users (using the observed age specific OC prevalence among hospital controls and female population size in the Oxford region).

The impact of possible differences between OCs containing levonorgestrel or a third generation progestogen (desogestrel or gestodene) with regard to stroke, AMI, and VTE risk were assessed using the same model

Table 1 Average mid-year population in Oxford region, total number of hospital controls interviewed, and prevalence (\%) of reported arterial disease risk factors, smoking, and OC use by age group

\begin{tabular}{|c|c|c|c|c|c|c|c|}
\hline \multirow[b]{3}{*}{ Age group } & \multirow{3}{*}{$\begin{array}{l}\text { Mid-year } \\
\text { population } \\
\text { (1000 women) }\end{array}$} & \multirow{3}{*}{$\begin{array}{l}\text { Total } \\
\text { hospital } \\
\text { controls }\end{array}$} & \multirow{3}{*}{$\begin{array}{l}\text { Hypertension, } \\
\text { diabetes or } \\
\text { hyperlipidaemia }\end{array}$} & \multicolumn{4}{|c|}{ No hypertension, diabetes or hyperlipidaemia } \\
\hline & & & & \multicolumn{2}{|c|}{ Non-smoker } & \multicolumn{2}{|l|}{ Smoker } \\
\hline & & & & Non-user & OC user & Non-user & OC user \\
\hline $20-24$ y & 101.5 & 139 & 2.2 & 18.0 & 37.4 & 11.5 & 30.9 \\
\hline $25-29 y$ & 107.9 & 122 & 7.4 & 29.5 & 27.9 & 14.8 & 20.5 \\
\hline $30-34 \mathrm{y}$ & 97.6 & 103 & 6.8 & 34.0 & 17.5 & 30.1 & 11.7 \\
\hline $35-39 y$ & 90.4 & 111 & 6.3 & 40.5 & 12.6 & 36.0 & 4.5 \\
\hline $40-44 y$ & 96.3 & 174 & 9.2 & 59.2 & 2.3 & 28.7 & 0.6 \\
\hline Total & 493.7 & 649 & 6.5 & 37.6 & 18.8 & 23.9 & 13.3 \\
\hline Age standardised $\star$ & & & 6.3 & 35.8 & 20.0 & 23.7 & 14.1 \\
\hline
\end{tabular}

* Standardised on age distribution of women aged $20-44$ years in Oxford region. 
Table 2 Estimated incidence of first hospitalisation for cardiovascular disease (per 100000 woman years) among all women and women who did not use OCs, smoke nor had other risk factors for arterial disease *

\begin{tabular}{|c|c|c|c|c|c|c|c|c|}
\hline \multirow[b]{2}{*}{ Age group } & \multicolumn{2}{|c|}{$\begin{array}{l}\text { Haemorrhagic stroke } \\
\text { (total } 62 \text { cases) }\end{array}$} & \multicolumn{2}{|c|}{$\begin{array}{l}\text { Ischaemic stroke } \\
\text { (total } 16 \text { cases) }\end{array}$} & \multicolumn{2}{|c|}{$\begin{array}{l}\text { Myocardial infarction } \\
\text { (total } 51 \text { cases) }\end{array}$} & \multicolumn{2}{|c|}{$\begin{array}{l}\text { Venous } \\
\text { thromboembolism } \\
\text { (total } 160 \text { cases) }\end{array}$} \\
\hline & $\begin{array}{l}\text { All } \\
\text { women }\end{array}$ & $\begin{array}{l}\text { Non-user } \\
\text { non-smoker }\end{array}$ & $\begin{array}{l}\text { All } \\
\text { women }\end{array}$ & $\begin{array}{l}\text { Non-user } \\
\text { non-smoker }\end{array}$ & $\begin{array}{l}\text { All } \\
\text { women }\end{array}$ & $\begin{array}{l}\text { Non-user } \\
\text { non-smoker }\end{array}$ & $\begin{array}{l}\text { All } \\
\text { women }\end{array}$ & Non-user \\
\hline $20-24 \mathrm{y}$ & 2.82 & 1.65 & 0.55 & 0.30 & 0.25 & 0.02 & 9.61 & 3.34 \\
\hline $25-29 y$ & 2.32 & 1.30 & 0.77 & 0.42 & 0.48 & 0.05 & 9.50 & 3.99 \\
\hline $30-34 \mathrm{y}$ & 4.02 & 2.27 & 0.85 & 0.47 & 1.58 & 0.20 & 6.91 & 3.94 \\
\hline $35-39 y$ & 5.53 & 3.25 & 0.92 & 0.54 & 1.70 & 0.28 & 8.02 & 5.79 \\
\hline $40-44 \mathrm{y}$ & 8.16 & 4.89 & 1.44 & 0.86 & 9.59 & 2.30 & 6.23 & 5.82 \\
\hline Age standardised $\dagger$ & 4.49 & 2.62 & 0.90 & 0.51 & 2.65 & 0.56 & 8.10 & 4.53 \\
\hline
\end{tabular}

*Incidence adjusted for estimated number of cases not included in the study (HS 30\%, IS $10 \%$, AMI 35\%, VTE 0\%) and estimated assuming the following risk ratios: HS: arterial disease risk factor 5, smoking 2, OC use 1.2; IS: arterial disease risk factor 5, smoking 2, OC use 1.3; AMI: arterial disease risk factor 5, smoking 10, OC use 3; VTE: levonorgestrel 2.6, third generation 5.4, other OC 3.1. †Standardised on age distribution of women aged 20-44 years in Oxford region.

with the same risk of HS, a range of risk estimates for IS and AMI, and a twofold higher VTE risk.

\section{Results}

PREVALENCE OF CARDIOVASCULAR RISK FACTORS About $6 \%$ of women had a reported history of the arterial disease risk factors hypertension, diabetes and hyperlipidaemia, the prevalence increasing with age (table 1). Among the remainder, over one third of women smoked, though the prevalence of smoking decreased with age, as did the prevalence of OC use. Among women in their 20s, OC use was greater among smokers (65\%) than nonsmokers (58\%) with the converse among women in their 30 s $(20 \%$ among smokers and $29 \%$ among non-smokers). The proportion of OC users who used OCs containing desogestrel or gestodene was approximately 34\% overall and dropped from 38\% among women aged $20-24$ years to $21 \%$ among women aged over 35 years. The low oestrogen dose levonorgestrel containing OCs were the most commonly used OC type (about $50 \%$ overall).

INCIDENCE OF TOTAL (NON-FATAL AND FATAL) CARDIOVASCULAR EVENTS

Incidence of HS among all women rose with age and was approximately five times more frequent than IS at all ages (table 2). AMI incidence rose more steeply with age than HS or IS among all women as well as those who did not use OCs, smoke nor had other cardiovascular risk factors. For all three diseases, the rise in incidence with age was approximately exponential. Age specific incidence of VTE among all women (users and non-users combined) decreased with age as did the prevalence of OC use (table 2 and figure 1). However the incidence among non-users increased linearly by approximately 1 event per 150000 woman years in each five year age group.

Total cardiovascular disease incidence among women who did not use OCs, smoke nor had other risk factors was modelled from the smoothed age specific incidence rates (table 3 and figure 2). It rose from 4.8 per 100000 woman years at age 20-24 years to 13.5 per 100000 woman years at ages $40-44$ years. At all ages, VTE was the most common cardiovascular event, representing two thirds of total cardiovascular incidence among women aged 20-24 years, but $44 \%$ among women aged 40-44.

Among users of low oestrogen dose OCs, VTE incidence was increased by a factor of 2.6 compared with non-users and represented, among users who did not smoke, $81 \%$ of all cardiovascular events for women aged 20-24 years (incidence 8.4 out of 10.3 per 100000 woman years) falling to $75 \%$ (11.9 out of 16.0 ) among women aged $30-34$, and 54\% (15.4 out of 28.4) among women aged 40-44 years. Overall, VTE represented $76 \%$ of all cardiovascular events among OC users who did not smoke (age standardised incidence 10.1 out of 13.3 per 100000 women years). The OC attributable cardiovascular events among nonsmokers were 5.53 per 100000 users annually at ages 20-24 years and rose to 14.9 per 100000 users annually at ages 40-44 years. Among non-smokers, $93 \%$ the OC attributable cardiovascular events were a result of VTE at ages $20-24$ years and $64 \%$ at ages $40-44$ years.

Among smokers who do not use OCs, the incidence of VTE was unchanged but stroke and AMI incidence were increased. The smoking attributable cardiovascular events were 1.70 per 100000 smokers annually at ages 20-24 years and rose to 24.6 per 100000 smokers annually at ages $40-44$ years.

Among low oestrogen dose OC users who smoked, total cardiovascular incidence rose from 12.6 per 100000 women annually at ages 20-24 years to 92.4 per 100000 women annually at ages $40-44$ years. In women age $20-24$ years the increase in risk compared with non-users who did not smoke was primarily because of an increase in VTE incidence, while in older women the increased incidence of stroke and AMI was the largest contributor.

INCIDENCE OF FATAL CARDIOVASCULAR EVENTS Total incidence of fatal cardiovascular events among non-smokers was low at all ages, whether OC users or not (table 3 and figure 3 ). HS and AMI represented $80 \%-90 \%$ of all cardiovascular deaths in each five year age group among non-users and $75 \%-90 \%$ among low oestrogen dose OC users. Among smokers, age specific cardiovascular death rates were dominated by $\mathrm{HS}$ and $\mathrm{AMI}$, and among OC users who smoked these two diseases accounted for $81 \%$ of cardiovascular deaths (HS 


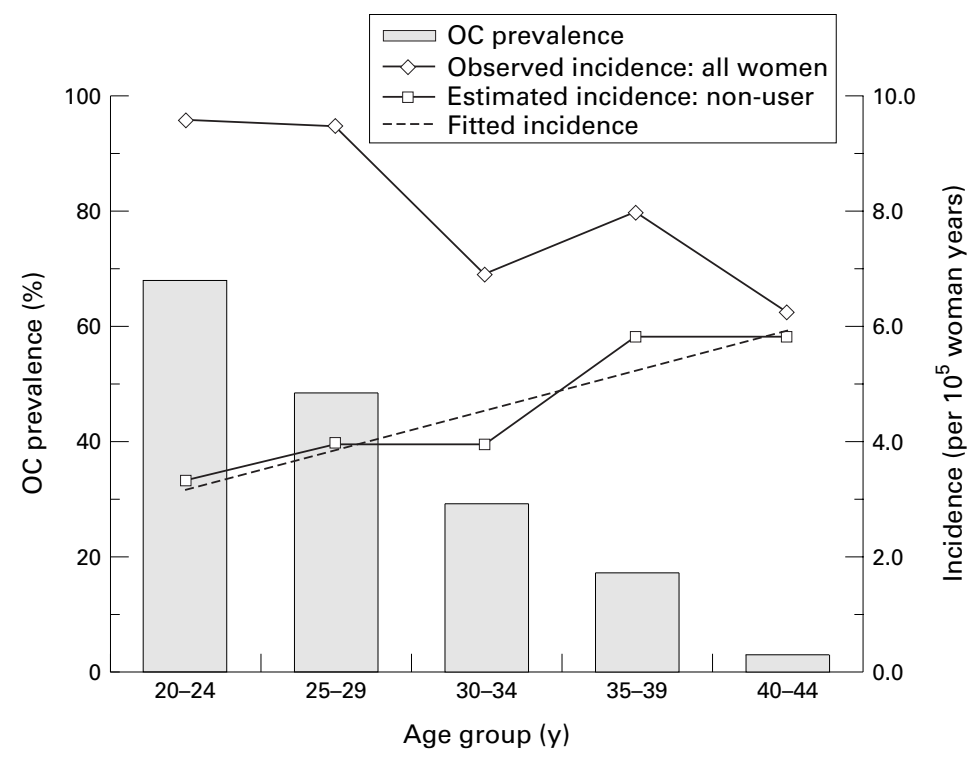

Figure 1 Age specific prevalence of $O C$ use and VTE incidence among all women and non-OC users.
Among women aged less than 35 years who did not smoke the age standardised incidence of fatal events per 100000 woman years was 1.0 among non-users and 1.4 among OC users. Corresponding figures among smokers were 2.2 and 3.2 per 100000 woman years. The additional risk attributable to OC use was 1 per 270000 non-smoking users and 1 per 98000 users who smoked.

Among women aged over 35 years who did not smoke the incidence of fatal events per 100000 woman years was 2.5 among nonusers and 3.8 among OC users, and, for smokers, 7.9 and 16.5, respectively. Additional risks attributable to OC use were 1.4 per 100000 non-smoking users, and 8.7 per 100000 users who smoked. The combination of smoking and OC use resulted in an additional 14 deaths per 100000 women annually.

\section{Discussion}

This simple model comparing total cardiovascular incidence and mortality is based on observed disease incidence rates in the Oxford region and estimates of relative risk from the WHO Collaborative Study. It is the first model to specifically incorporate the effects of age, smoking and use of low oestrogen dose combined OCs on all cardiovascular diseases and is based on estimates of relative risk and disease incidence rates from a study conducted in the early 1990s. It shows that a full comparison of OC associated risks must consider age specific incidence and mortality of all four diseases (HS, IS, AMI, and VTE). Total incidence of all fatal cardiovascular events associated with OC use among young women without cardiovascular risk factors was low and the small risk associated with OC use was substantially less than the risk associated with smoking. The combination of smoking and OC use, particularly at older ages, was associated with a substantial increase in risk-1 additional death per 3000 woman years for OC users aged 40-44 years who smoke. Mortality was 30-34, and 40-44 years, respectively.

Table 3 Modelled incidence of total and fatal cardiovascular events (per 100000 woman years) for low oestrogen dose OC users and smokers*

\begin{tabular}{|c|c|c|c|c|c|c|c|c|c|c|c|}
\hline \multirow[b]{3}{*}{ Age group } & \multicolumn{6}{|c|}{ Non-smoker } & \multicolumn{2}{|l|}{ Smoker } & \multicolumn{3}{|c|}{ Attributable risk } \\
\hline & \multicolumn{5}{|c|}{ Non-user } & \multirow[b]{2}{*}{ OC user } & \multirow[b]{2}{*}{ Non-user } & \multirow[b]{2}{*}{ OC user } & \multirow[b]{2}{*}{ OC use } & \multirow[b]{2}{*}{ Smoking } & \multirow{2}{*}{$\begin{array}{l}\text { OC use } \\
\text { and } \\
\text { smoking }\end{array}$} \\
\hline & $H S$ & IS & $A M I$ & $V T E$ & Total & & & & & & \\
\hline \multicolumn{12}{|l|}{ Total events } \\
\hline $20-24$ y & 1.27 & 0.30 & 0.01 & 3.22 & 4.81 & 10.3 & 6.51 & 12.6 & 5.53 & 1.70 & 7.81 \\
\hline $25-29 y$ & 1.76 & 0.39 & 0.05 & 3.90 & 6.09 & 12.9 & 8.66 & 16.8 & 6.80 & 2.57 & 10.7 \\
\hline $30-34 \mathrm{y}$ & 2.43 & 0.49 & 0.17 & 4.58 & 7.66 & 16.0 & 12.1 & 24.1 & 8.29 & 4.45 & 16.4 \\
\hline $35-39 \mathrm{y}$ & 3.35 & 0.63 & 0.60 & 5.25 & 9.83 & 20.3 & 19.2 & 41.4 & 10.5 & 9.39 & 31.5 \\
\hline $40-44$ y & 4.63 & 0.80 & 2.13 & 5.93 & 13.5 & 28.4 & 38.1 & 92.4 & 14.9 & 24.6 & 79.0 \\
\hline \multicolumn{12}{|c|}{ Age standardised $\dagger$} \\
\hline Population & 2.64 & 0.51 & 0.57 & 4.54 & 8.27 & 17.4 & 16.6 & 36.6 & 9.09 & 8.30 & 28.4 \\
\hline OC users & 1.87 & 0.40 & 0.14 & 3.89 & 6.29 & 13.3 & 9.81 & 19.8 & 7.00 & 3.52 & 13.5 \\
\hline \multicolumn{12}{|l|}{ Fatal events } \\
\hline $20-24$ y & 0.64 & 0.09 & 0.01 & 0.06 & 0.80 & 1.07 & 1.58 & 2.12 & 0.27 & 0.78 & 1.32 \\
\hline $25-29 y$ & 0.88 & 0.12 & 0.02 & 0.08 & 1.09 & 1.47 & 2.29 & 3.27 & 0.38 & 1.19 & 2.18 \\
\hline $30-34 \mathrm{y}$ & 1.21 & 0.15 & 0.08 & 0.09 & 1.53 & 2.12 & 3.59 & 5.88 & 0.59 & 2.06 & 4.35 \\
\hline $35-39 y$ & 1.68 & 0.19 & 0.28 & 0.11 & 2.25 & 3.36 & 6.60 & 13.1 & 1.11 & 4.35 & 10.8 \\
\hline $40-44$ y & 2.32 & 0.24 & 0.98 & 0.12 & 3.65 & 6.34 & 15.0 & 35.9 & 2.68 & 11.4 & 32.2 \\
\hline \multicolumn{12}{|c|}{ Age standardised $\dagger$} \\
\hline Population & 1.32 & 0.15 & 0.26 & 0.09 & 1.83 & 2.81 & 5.67 & 11.7 & 0.98 & 3.84 & 9.87 \\
\hline OC users & 0.93 & 0.12 & 0.06 & 0.08 & 1.19 & 1.67 & 2.82 & 4.67 & 0.48 & 1.63 & 3.48 \\
\hline
\end{tabular}

${ }^{\star}$ Modelled assuming the following risk ratios: HS (haemorrhagic stroke): smoking 2, OC use 1.2; IS (ischaemic stroke): smoking 2 , OC use 1.3; AMI (acute myocardial infarction): smoking 10, OC use 3; VTE (venous thromboembolism): OC use 2.6. †Standardised on age distribution of all women aged 20-44 years in Oxford region, or age distribution of OC users. 
strongly influenced by smoking and dominated by arterial diseases (HS and AMI, in particular) the incidence of which rose exponentially with age. However, among non-smokers or younger women, OC associated mortality was very low and the additional VTE events associated with OC use were comparatively frequent, even though VTE case fatality is low and nonfatal VTE events less debilitating than other non-fatal, acute cardiovascular diseases.

The WHO Study indicated the importance of screening for cardiovascular risk factors, in particular detection of increased blood pressure, before OC use to minimise any OC associated risk. Among non-smoking women with no other cardiovascular risk factors who reported a blood pressure check before OC use, the relative risk estimate for AMI was 1.1 (95\% confidence intervals $0.2,5.7),{ }^{5}$ compatible with recent results from a case-control study conducted among women participating in a managed healthcare programme in California

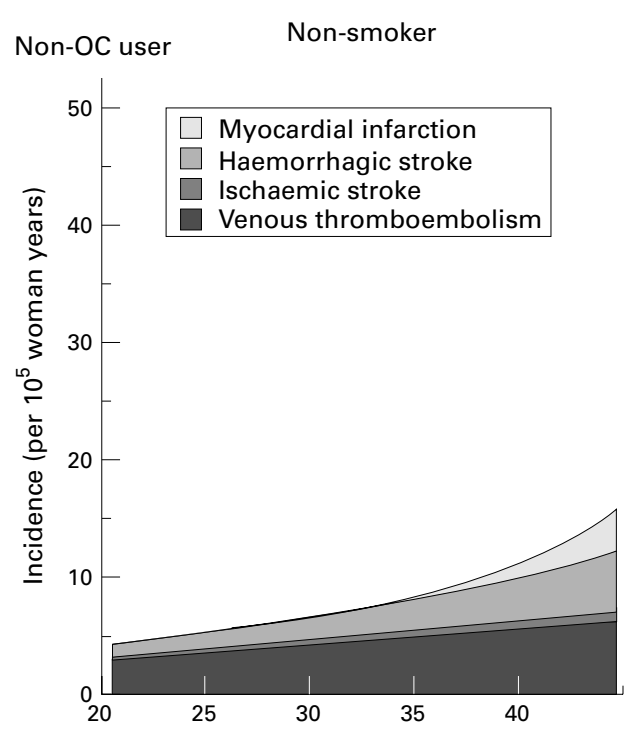

Low oestrogen dose OC user

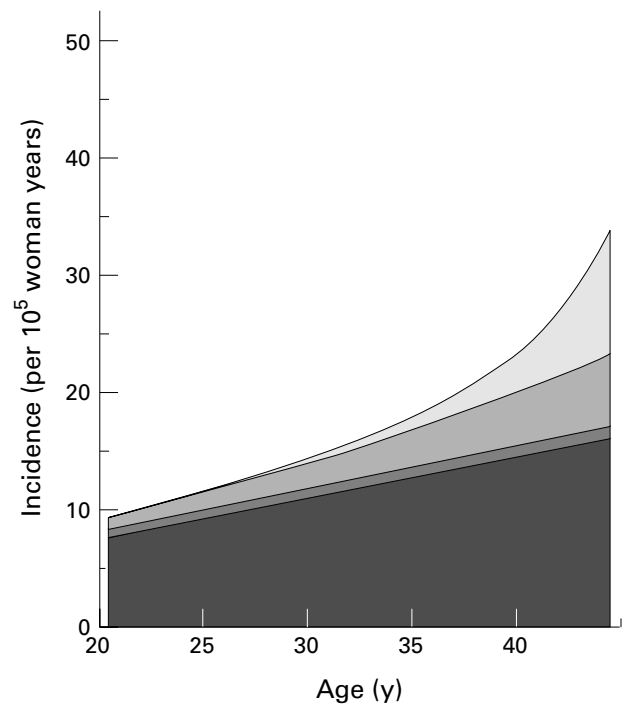

that showed at most a small increase in risk. ${ }^{12}$ Similarly, IS risk was about $50 \%$ lower among women who reported blood pressure checking before OC use, ${ }^{3}$ and among such women risk estimates were in accordance with results from the same study in the USA of ischaemic and haemorrhagic stroke risks associated with OC use. ${ }^{13}$ Both studies showed little impact of OCs on HS among younger women, but a doubling in risk among women over age 35 years. $^{4}$ Applying such risks in the model (HS risk 1.0 for women aged 20-34 years and 2.0 for those aged $\geqslant 35$ years, AMI risk 1.1 and IS risk 1.2 at all ages) did not change the incidence of total (non-fatal and fatal) cardiovascular events in table 3 among non-users (smokers and/or nonsmokers) and had a small impact on incidence rates among users who did or did not smoke (age standardised incidence reduced from 19.8 to 17.1 and 13.3 to 13.0 per 100000 woman years among smokers and non-smokers, respectively). However, the impact on fatal
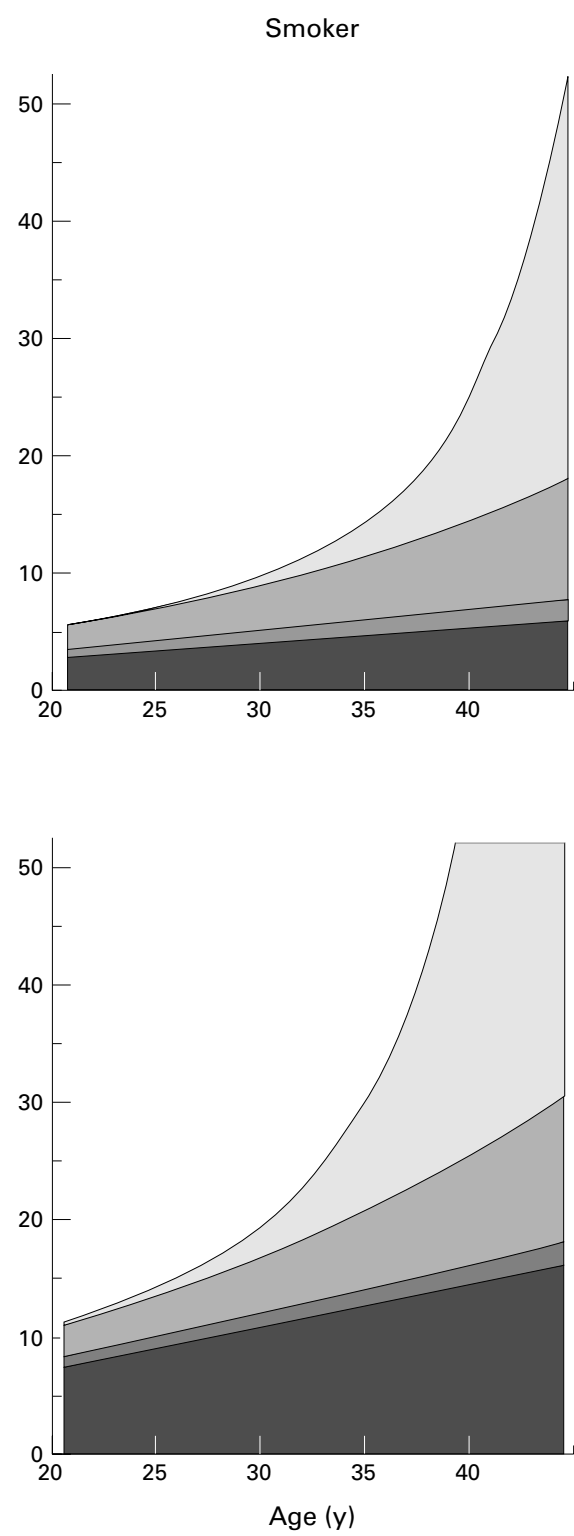

Figure 2 Modelled incidence of first hospitalisation for cardiovascular disease (per 100000 woman years) according to $O C$ use and smoking. 
events was greater. The age standardised mortality in OC users was 1.5 per 100000 woman years among non-smokers and 3.4 per 100000 woman years among smokers, corresponding to attributable risks of 1 per 300000 woman years and 1 per 44000 users annually, respectively. Among non-smokers aged less than 35 years the risk of death attributable to OC use was approximately 1 per 700000 among users who had been screened annually.

These OC attributable risks must be seen in the context of other risks to which healthy women of reproductive age are exposed. All cause mortality rates in England and Wales in 1991 and $1992^{14}{ }^{15}$ were $31.1,43.5$, and 106.5 per 100000 woman years in women at ages 15-24, 25-34, and 35-44 years, respectively. Deaths attributable to external causes (accidents, injuries, poisoning, suicide or homicide (ICD 9th revision codes E800-E999)) are more stable with age and average 13.1 per 100000 woman years in the age range 15-44

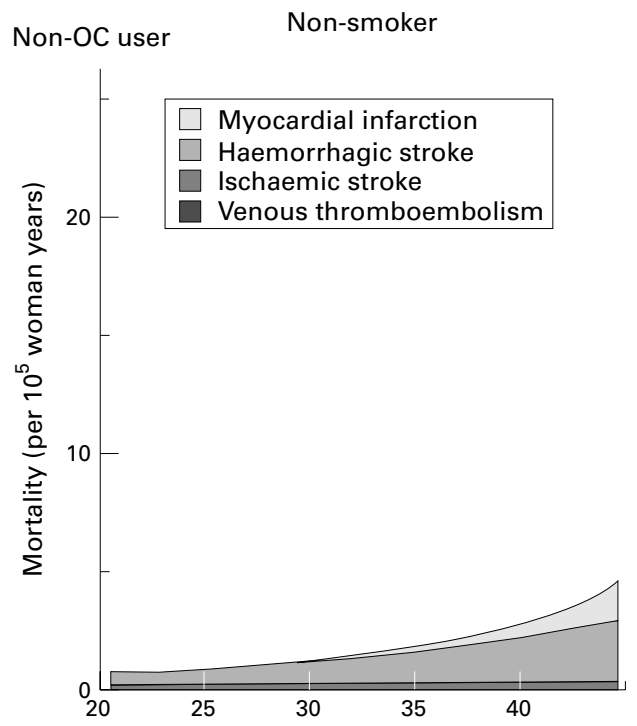

Low oestrogen dose OC user

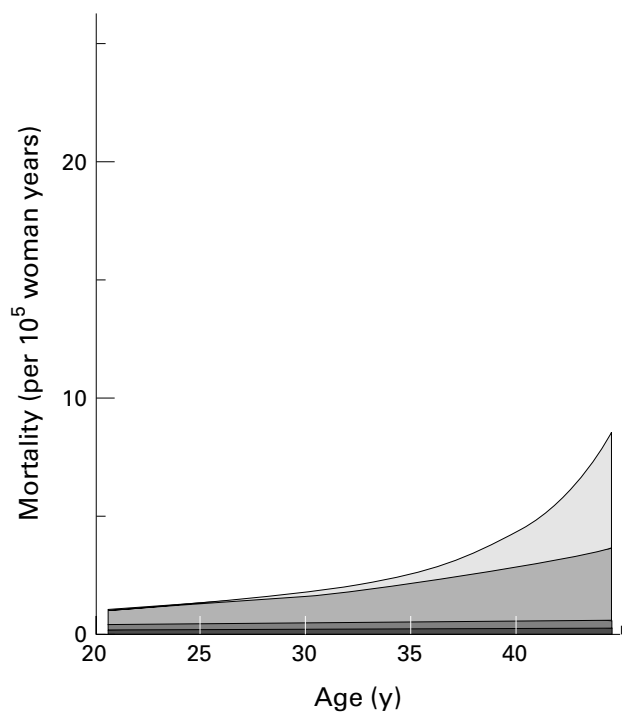

years. Considering the substantial benefits of using a reliable, reversible contraceptive method, estimated OC attributable risks less than 1 per 100000 users annually (for example, in non-smokers under age 35 years) or about 2 per 100000 users annually (for example, in smokers under age 35 years) appear trivial.

VALIDITY OF OBSERVED INCIDENCE RATES

Overall incidence rates were computed from the relatively small numbers of cases identified in the Oxford region, and may have been subject to considerable error. However, the estimated disease incidence rates among all women correspond closely with the reported cardiovascular death rates in the Oxford region during the period of the study.

Deaths from acute myocardial infarction (ICD 9th revision code 410) were 1.0 and 2.7 per 100000 women annually at ages 25-34 and 35-44 years, or 1.81 per 100000 women
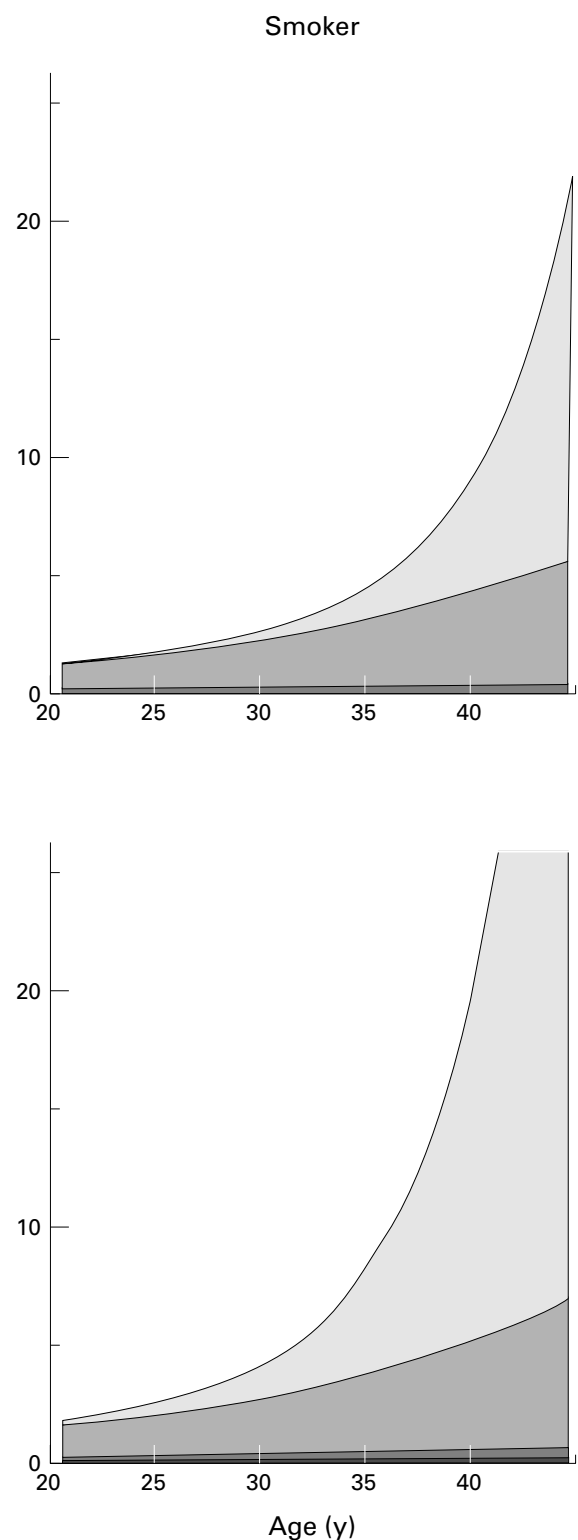

Figure 3 Modelled incidence of fatal first cardiovascular event (per 100000 woman years) according to OC use and smoking. 
annually at age $25-44$ years. $^{8}$ The corresponding incidence of AMI (including fatal and nonfatal events) in the WHO study (table 2) were 1.0 and 5.7 per 100000 women annually at ages 25-34 and 35-44 years, or 3.27 per 100000 women annually at age $25-44$ years. The ratio of these rates $(55 \%)$ is close to the assumed $46 \% 28$ day case fatality rate based on results from the MONICA study. ${ }^{10}$

Similarly the stroke death rates (ICD 9th revision codes 430-438) were 1.2 and 4.7 per 100000 women annually at ages $25-34$ and 35-44 years, or 2.87 per 100000 women annually at age 25-44 years in the Oxford region. ${ }^{8}$ The corresponding stroke incidence rates in the WHO study (haemorrhagic and ischaemic stroke combined) (table 2) were 3.94 and 8.07 per 100000 women annually at ages 25-34 and 35-44 years, or 5.91 per 100000 women annually at age 25-44 years. The ratio of these rates $(49 \%)$ is close to the $46 \% 28$ day stroke case fatality rate computed from the MONICA study ${ }^{9}$ assuming a ratio of four haemorrhagic to one ischaemic stroke case.

A total of four VTE deaths (ICD-9 codes 451-453) were recorded in women age 25-44 years in the Oxford region during period of the study (0.24 per 100000 woman years). ${ }^{8}$ This compares with a total of 121 idiopathic VTE cases (7.71 per 100000 woman years) recorded in the study in this age group (table 2) and the assumed $2 \% 28$ day case fatality rate. ${ }^{11}$ It should be noted that the idiopathic cases considered in the WHO study excluded any events associated with pregnancy, recent surgery or prolonged bed rest.

VALIDITY OF MODEL AND SENSITIVITY TO ASSUMPTIONS

As the cardiovascular disease incidence and death rates arose from a limited population base, covering 2 million woman years of observation, it is important to consider other sources of information that support the assumptions on which the model is based.

The exponential rise in stroke and AMI incidence with age have support from other sources, including the recent study in the USA,${ }^{16}$ national mortality statistics, ${ }^{14}{ }^{15}$ and the MONICA project. ${ }^{9}{ }^{10}$ Although these statistics include all events, not just first events among women free of arterial disease risk factors, the age specific patterns of disease in the Oxford region were similar. ${ }^{8}$ Thus the exponential smoothing of risk estimates did not introduce any substantial error. The overall VTE incidence among non-users and users of second and third generation OCs was close to that reported in UK general practices ${ }^{17}$ and the age specific rise in incidence was also reported by Farmer and colleagues. ${ }^{18}$ These authors reported an exponential increase with age but included less well validated cases. Nevertheless an exponential model fitted to the estimated VTE incidence (fig 1) resulted in almost identical conclusions. Although the age specific VTE incidence rates among all except pregnant and puerperal women in Denmark ${ }^{19}$ are higher than those observed in the Oxford
KEY POINTS

- Incidence of cardiovascular diseases (idiopathic venous thromboembolism, stroke, and acute myocardial infarction) is very low among women of reproductive age.

- Incidence of idiopathic venous thromboembolism rises less steeply with age than acute myocardial infarction or stroke.

- Mortality associated with combined oral contraceptive use is low among women aged less than 35 years, even if they smoke.

- Among healthy women over 35 years, the additional mortality associated with oral contraceptive use is 1.4 per 100000 users per year, less than that associated with smoking (5.4 per 100000 smokers per year) or the combination of the two (14 per 100000 OC users who smoke per year)

- Although idiopathic venous thromboembolism causes less morbidity and mortality than stroke or acute myocardial infarction, it is the most common cardiovascular side effect of oral contraceptive use among women in Europe

region, the data reported here refer only to idiopathic VTE and thus additionally exclude women with recent surgery, trauma or prolonged bed rest.

An earlier study conducted in England and Wales between 1986 and $1988^{20}$ reported 21 ischaemic ("occlusive") and 401 haemorrhagic stroke deaths - a ratio of 1:20. In contrast, in the WHO Study in the Oxford region the incidence of IS was approximately one fifth that for HS. This ratio included both fatal and non-fatal events, and was approximately $1: 8$ for fatal strokes when the assumed 28 day case fatality rates for IS and HS were considered. ${ }^{9}$ These ratios were based on small numbers of cases and were lower than the more commonly reported 1:2 ratio of IS to HS among older women ${ }^{21}$ or results from the recent case-control study among reproductive age women in California that reported similar numbers of IS and HS cases and an overall stroke incidence of 11.3 per 100000 woman years. ${ }^{13} \mathrm{~A}$ recent population-based study of stroke among reproductive age women in Washington State, USA $^{22}$ reported average incidence rates of 4.3 and 6.4 per 100000 woman years for ischaemic and haemorrhagic stroke, respectively. While differences in healthcare setting, prevalence and treatment of cardiovascular disease risk factors, and smoking rates may partially explain the discrepancy with the Oxford region, arbitrarily multiplying IS incidence at all ages by 5 (so that total age standardised stroke incidence was approximately 10 per 100000 woman years) did not change the main implications of the model-OC associated cardiovascular mortality was dominated by the risk of arterial diseases, which are more important among older women and those who smoke, and the excess 
VTE risk associated with OC use was the largest contributor to the incidence of total cardiovascular events among non-smokers and smokers aged less than 35 years.

The estimated incidence for VTE and IS among non-users who did not smoke were similar to those computed for a Canadian population, ${ }^{23}$ though estimated AMI incidence was lower than in the Canadian population, particularly among younger women. However, the method of estimation used for the WHO data was based on fewer assumptions. The low hospitalisation rates for all four cardiovascular diseases observed in the WHO Study may partly reflect the specificity of the diagnostic criteria, the fact that only first events were considered, and the exclusion of women with conditions predisposing to VTE, such as recent surgery, trauma, prolonged bed rest or pregnancy. The AMI incidence rates among women in the Oxford region were closer to those observed in the managed healthcare programme in California ${ }^{16}$ though they rose less steeply with age.

The prevalence and patterns of OC use and smoking were estimated from hospital controls rather than the general population and may not reflect the true rates in the UK or Oxford region. However, national rates of $\mathrm{OC} \mathrm{use}^{24}$ among young women-36\% at ages $25-34$ years and $12 \%$ at ages $35-44$ years-were similar to those reported here $(39 \%$ and $10 \%$, respectively), but, as expected, national rates of smoking $^{24}$ were slightly lower-33\% and $28 \%$ in the two age groups, respectively, compared with $38 \%$ and $35 \%$, respectively, observed among the hospital controls. The proportion of OC users who smoked may also have been overestimated in the hospital controls than in the general population, though the estimates of stroke and AMI incidence rates among non OC users who did not smoke nor had other risk factors for cardiovascular disease were little changed when slightly different patterns of smoking and OC use were assumed.

The magnitude of the risk ratios assumed for different OC preparations may also differ from the true values, but there has been confirmation of the WHO results on VTE from independent sources ${ }^{17} 182526$ and risk estimates assumed for IS, HS, and AMI are compatible with earlier reports. ${ }^{27}$ As the prevalence of diagnosed major arterial disease risk factors other than smoking (hypertension, diabetes, and hyperlipidaemia) was low at all ages, the estimation of incidence rates for IS, HS, and AMI among non-users without cardiovascular risk factors was little influenced by different assumptions on the magnitude of the stroke and AMI risks associated with these conditions.

Differences between the true and assumed case fatality rates for each disease would have a large impact on the model, in particular for the incidence of all fatal cardiovascular events as well as the estimation of incidence rates among non-users without cardiovascular risk factors. The VTE case fatality rate $(2 \%)$ was taken from an early review ${ }^{11}$ and there are few more recent data, though Jick et $a l^{17}$ observed six fatal and 80 non-fatal hospitalised VTE cases in two similar cohorts of OC users. Any increase in VTE case fatality would not affect estimates of incidence of total cardiovascular events, but would make a greater contribution to the estimated incidence of fatal cardiovascular events, particularly among OC users. The case fatality rates for stroke and AMI are based on estimates from the MONICA study ${ }^{9}$ for women aged up to 65, and may overestimate the rates among women under age 45 years. Moreover, case fatality rates for AMI are strongly influenced by the intervention rate and the rapidity of transfer to a specialist unit, ${ }^{28}$ and the $46 \% 30$ day case fatality rate assumed here may be too high for reproductive aged women in the Oxford region during the period of the study. The estimated number of cases missed from the study was based on the same sources, considering the proportion of fatal cases who died within the first 24 hours of the event. Few better sources of such information have been found. If only one third of AMI cases are not hospitalised or die within the first 24 hours of admission then the AMI incidence rates may have been overestimated by $30-40 \%$ and the impact of AMI on OC associated mortality may have been exaggerated. However, the conclusions of the model would not be substantially different as these are dominated by the steep rise in AMI incidence with age and the major adverse effect of smoking. Similarly, it may be hypothesised that case fatality rates for cardiovascular disease differ according to the presence of risk factors or whether the patient smoked or used OCs, but we have found no data to quantify such differences. A recent report from the Auckland community-based register has shown that although in-hospital case fatality rates for smokers and non-smokers differ, the total case fatality rates are similar. ${ }^{29}$

The model assumed that stroke and AMI risks for women who used OCs and smoked was the product of the risks for smoking and OC use separately. Such a multiplicative model was based on the results from the WHO Study ${ }^{3-5}$ that showed more than additive effects of these factors and reasonable agreement with such a multiplicative model. The study was the largest and most recent to quantify risks associated with OC use and other risk factors, and the results are compatible with those from previous studies. If we had assumed additive risks, the estimated incidence rates among non-OC users who did not smoke nor have other risk factors would have been little different from those used in the model, but the large adverse impact of smoking and OC use would have been less. However, the main conclusions of the model would have been little changed because they are dominated by the very different patterns of venous and arterial diseases according to age and cardiovascular risk factors.

The model only considers the cardiovascular risks associated with $\mathrm{OC}$ use and does not incorporate risks of other potential adverse effects, such as on breast or cervical cancer, nor beneficial effects, such on as ovarian and endometrial cancer. While the model includes 
Table 4 Modelled incidence of total and fatal cardiovascular events (per 100000 woman years) for different types of OC user

\begin{tabular}{|c|c|c|c|c|c|c|c|c|}
\hline \multirow[b]{3}{*}{ Age group } & \multicolumn{4}{|c|}{ Non-smoker } & \multicolumn{4}{|l|}{ Smoker } \\
\hline & \multirow[b]{2}{*}{ Non-user } & \multirow[b]{2}{*}{ Levonorgestrel } & \multicolumn{2}{|c|}{ Third generation } & \multirow[b]{2}{*}{ Non-user } & \multirow[b]{2}{*}{ Levonorgestrel } & \multicolumn{2}{|c|}{ Third generation } \\
\hline & & & Low & High & & & Low & High \\
\hline \multicolumn{9}{|l|}{ Total events } \\
\hline $20-24$ y & 4.81 & 10.3 & 19.2 & 19.4 & 6.51 & 12.6 & 21.2 & 21.6 \\
\hline $25-29 y$ & 6.09 & 12.9 & 23.6 & 23.8 & 8.66 & 16.8 & 26.5 & 27.7 \\
\hline $30-34$ y & 7.66 & 16.0 & 28.3 & 28.8 & 12.1 & 24.1 & 33.2 & 36.9 \\
\hline $35-39 y$ & 9.83 & 20.3 & 33.6 & 35.0 & 19.2 & 41.4 & 43.7 & 56.1 \\
\hline $40-44$ y & 13.5 & 28.4 & 40.5 & 45.0 & 38.1 & 92.4 & 66.0 & 109.0 \\
\hline \multicolumn{9}{|c|}{ Age standardised $\dagger$} \\
\hline Population & 8.27 & 17.4 & 28.8 & 30.1 & 16.6 & 36.6 & 37.6 & 49.3 \\
\hline OC users & 6.29 & 13.3 & 23.8 & 24.2 & 9.81 & 19.8 & 27.7 & 30.7 \\
\hline \multicolumn{9}{|l|}{ Fatal events } \\
\hline $20-24$ y & 0.80 & 1.07 & 1.21 & 1.25 & 1.58 & 2.12 & 2.12 & 2.30 \\
\hline $25-29 y$ & 1.09 & 1.47 & 1.61 & 1.69 & 2.29 & 3.27 & 2.98 & 3.49 \\
\hline $30-34 \mathrm{y}$ & 1.53 & 2.12 & 2.18 & 2.38 & 3.59 & 5.88 & 4.48 & 6.13 \\
\hline $35-39 y$ & 2.25 & 3.36 & 3.04 & 3.65 & 6.60 & 13.1 & 7.73 & 13.4 \\
\hline $40-44$ y & 3.65 & 6.34 & 4.64 & 6.67 & 15.0 & 35.9 & 16.5 & 36.2 \\
\hline \multicolumn{9}{|c|}{ Age standardised $\dagger$} \\
\hline Population & 1.83 & 2.81 & 2.49 & 3.07 & 5.67 & 11.7 & 6.60 & 12.0 \\
\hline OC users & 1.19 & 1.67 & 1.72 & 1.89 & 2.82 & 4.67 & 3.54 & 4.89 \\
\hline
\end{tabular}

*Modelled assuming the following risk ratios: HS: smoking 2, Levonorgestrel 1.2, 3Gen-low 1.2, 3Gen-high 1.2; IS: smoking 2, Levonorgestrel 1.3, 3Gen-low 1.0, 3Gen-high 1.3; AMI: smoking 10, Levonorgestrel 3.0, 3Gen-low 1.0, 3Gen-high 3.0; VTE: Levonorgestrel 2.6, 3Gen-low 5.4, 3Gen-high 5.4. +Standardised on age distribution of women aged 20-44 years in Oxford region, or age distribution of OC users.

the number of cardiovascular deaths expected among groups of women according to smoking status and $\mathrm{OC}$ use, it does not attempt to weigh the comparative severity of non-fatal cardiovascular events. Such features could easily be incorporated into the model, but no data have been found to estimate the rates of severe disability among women who have suffered a stroke, AMI or VTE. However, the severe disability rates after stroke or AMI are much higher than for VTE. This pattern is similar to the relative death rates for the different diseases assumed in the model. Thus conclusions based on a model that included disability in addition to mortality would be similar to those based on the mortality component of the model considered here, but would not reflect the relative severity of disability caused by the different diseases.

RISKS IN OTHER POPULATIONS AND ACCORDING TO OC TYPE

The simple model presented here can be extended to populations with different patterns of cardiovascular disease and risk factors. ${ }^{30}$ For example, women with activated protein C (APC) resistance are at eightfold higher risk of VTE than others. ${ }^{31}$ Prevalence of Leiden factor $\mathrm{V}$ mutation is approximately $5 \%$ in the UK and other European countries, and almost absent among non-white populations. ${ }^{32} 33$ Assuming OC use is independent of carrier status and VTE risks associated OC use and carrier status are multiplicative,,$^{25} 31$ the estimated VTE incidence in a population with no APC resistance is $26 \%$ lower in all women, including OC users and non-users. As the contribution of VTE to total mortality is very small, patterns of cardiovascular deaths would be little different in a population with no APC resistance. However, the lower VTE incidence is of greater importance when considering incidence of all (non-fatal and fatal) cardiovascular events, particularly among younger women and those who do not smoke.
The model also provides a framework for assessing the impact of possible differences in risk according to type of $\mathrm{OC}$ on total and fatal cardiovascular events. Five epidemiological studies $^{217} 18^{25} 26$ have shown that third generation OCs are associated with a twofold higher VTE risk than low oestrogen dose levonorgestrel containing products, and a potential biological mechanism has been identified. ${ }^{35} \mathrm{Re}-$ sults from the Transnational study conducted in five European countries suggested a lower AMI risk among users of such products but this was not seen in the UK, which contributed five of the seven exposed cases. ${ }^{36}$ The finding has yet to be independently replicated, but if true, suggests that there might also be a lower risk of IS. This can be modelled by considering two extreme assumptions-third generation OCs carry no increased risk of IS or AMI compared with non-users ( 3 Gen-Low) or the same IS and AMI risk as the levonorgestrel products ( 3 Gen-High) (table 4 and fig 4). Among nonsmokers, the incidence of total (non-fatal and fatal) cardiovascular events among users of third generation OCs is higher than for levonorgestrel OCs at all ages, but the range of death rates for third generation products includes the estimated rate for levonorgestrel OCs from age 35 years onwards. However, the age standardised death rates are very similar (1.7-1.9 per 100000 woman years). Among smokers, third generation OCs might confer an advantage from around age 35 years for total cardiovascular events or from around age 25 years for fatal events. Among OC users who smoke the difference in mortality according to OC type is at most 1 per 100000 woman years among all users, and 1 per 250000 woman years among those aged less than 35 years. At all ages cardiovascular mortality among smokers is higher than that among OC users, even under the most extreme assumptions regarding patterns of risk according to OC type.

These findings that the excess VTE deaths associated with use of third generation OCs 
might be offset by a lower incidence of fatal AMIs are similar to those reached for groups of OC users from Canada ${ }^{37}$ and USA, ${ }^{38}$ though neither of these reports explicitly modelled the impact of smoking. Our model demonstrates the importance of considering age of the user and her smoking status when attempting to balance any potential trade off between an excess VTE and lower IS and AMI risk.

\section{CONCLUSION}

The data and model presented here show that cardiovascular mortality associated with OC use among apparently healthy women less than 35 years old who do not smoke is very low and in the order of 1 death per 250000 users annually. The data from the WHO Study also indicate that if such women are screened for cardiovascular risk factors, OC associated mortality may be less than half this rate. Risks associated with smoking, even among non-OC users, are substantially greater. However, many young women smoke and have a need for a

\section{Total events}

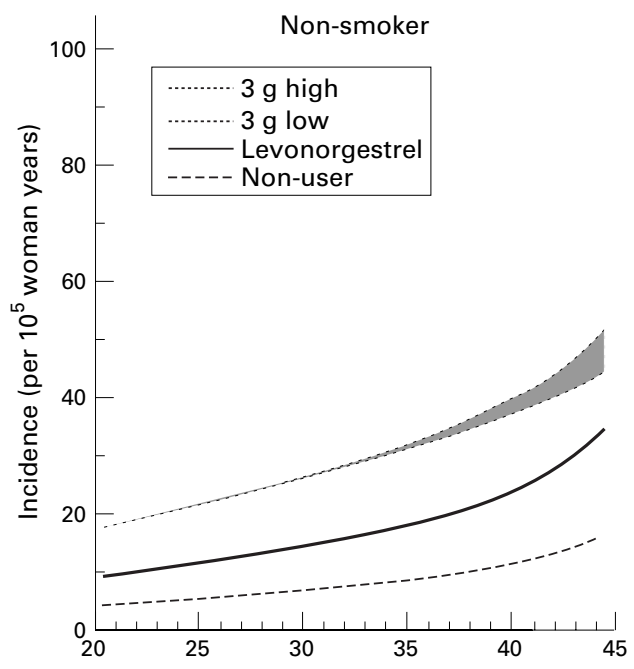

Fatal events

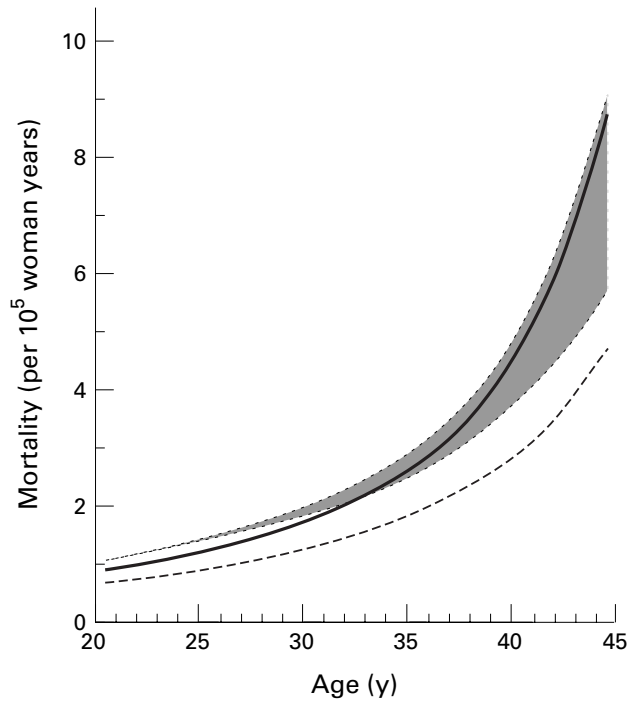

simple, reliable, and reversible contraceptive method-the mortality attributable to OC use among women less than 35 years who smoke is estimated to be about 1 per 100000 users annually. Among women above the age of 35 years who smoke and use OCs, the additional risk of death is much higher (about 1 per 7000 users who smoke annually).

The adverse impact of OCs on VTE risk has little influence on mortality, but constitutes a substantial portion of OC associated cardiovascular events, in particular among women less than 35 years of age, even with the low oestrogen dose levonorgestrel OCs that carry less VTE risk than the third generation products. These data reinforce the importance of providing adequate information to current and prospective OC users about OC associated cardiovascular risks and their interaction with smoking and other cardiovascular risk factors. Together with the results from the WHO Study, they underline the benefit of screening for risk factors, particularly among older
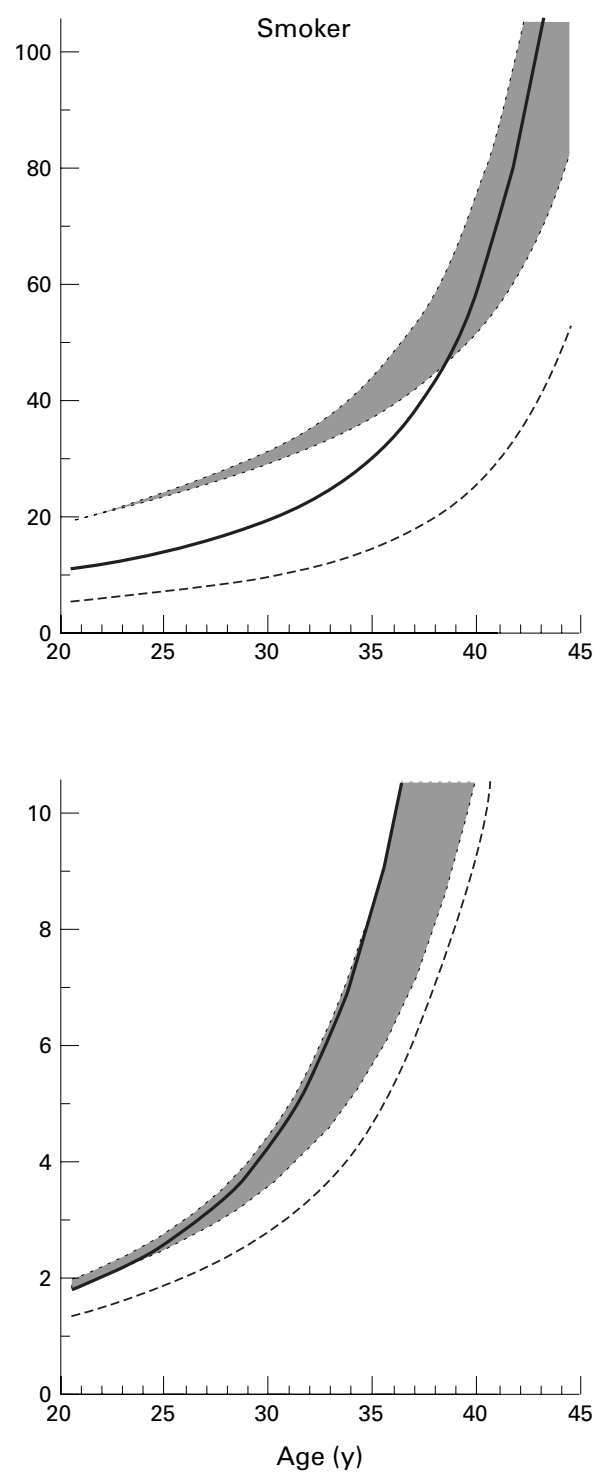

Figure 4 Modelled incidence of total and fatal cardiovascular event (per 100000 woman years) by type of OC user. Shaded area represents range of plausible values for third generation products. 
women, to minimise adverse cardiovascular effects of OC use.

The avoidable excess VTE risk with third generation OCs accounts for a substantial proportion of total cardiovascular events, though this must be weighed against the substantially lower morbidity associated with non-fatal VTE compared with stroke or AMI. For women over 35 years, among whom the burden of arterial diseases is higher, any difference in AMI risk between products would be a more important consideration, particularly among smokers. OC users who smoke might consider that any potential reduction in AMI risk associated with the new products balances the reported excess VTE risk, though users who are worried about cardiovascular side effects would be better advised to stop smoking or switch to a different contraceptive method. Choice between OC brands should not only be made on what are at most very small differences in cardiovascular mortality but must also consider other factors, including differences in non-fatal cardiovascular events and personal preferences.

The widespread use of OCs by young healthy women for protection against pregnancy means that the threshold at which side effects will be considered acceptable is low and the public perception of risks often exaggerated. Nevertheless, the choice should be made with the knowledge of the reported risks and benefits of different contraceptive methods.

The data used in this report draw on those collected under the supervision of Professor Martin Vessey in the Oxford region, UK, as part of the WHO Collaborative Study of Cardiovascular Disease and Steroid Hormone Contraception. We thank Soledad Diaz, Anna Glasier, Barbara Hulka, Joe Kelaghan, David Skegg, and Margaret Thorogood who provided valuable comments to this report and Michael Marmot for continued advice and encouragement throughout the study.

The views presented here are those of the authors and do not necessarily reflect those of the World Health Organization.

Funding: The WHO Collaborative Study of Cardiovascular Disease and Steroid Hormone Contraception, was funded by the UNDP/UNFPA/WHO/World Bank Special Programme of Research, Development and Research Training in Human Reproduction and the National Institutes of Health Contracep-
tive and Reproductive Evaluation Branch (contract NICHD NO1-HD-0-2914).

Conflicts of interest: none.

1 World Health Organization Collaborative Study of Cardiovascular Disease and Steroid Hormone Contraception. Venous thromboembolic disease and combined oral contraceptives: Results of an international multicentre case-control study. Lancet 1995;346:1575-82 .

2 World Health Organization Collaborative Study of Cardiovascular Disease and Steroid Hormone Contraception. Effect of different progestagens in low oestrogen oral contraceptives on venous thromboembolic disease. Lancet 1995;346:1582-8.

3 World Health Organization Collaborative Study of Cardiovascular Disease and Steroid Hormone Contraception. Ischaemic stroke and combined oral contraceptives: Ischaemic stroke and combined oral contraceptives:
Results of an international multicentre case-control study. Results of an international
Lancet 1996;348:498-505.

4 World Health Organization Collaborative Study of Cardiovascular Disease and Steroid Hormone Contraception Haemorrhagic stroke, overall stroke risk, and combined oral contraceptives: Results of an international, multicentre, case-control study. Lancet 1996;348:505-10.

5 World Health Organization Collaborative Study of Cardiovascular Disease and Steroid Hormone Contraception. Acute myocardial infarction and combined oral contraceptives: Results of an international multicentre case-control study. Lancet 1997;349:1202-9.

6 Poulter NR, Chang CL, Farley TMM, et al. Reliability of data from proxy respondents in an international casecontrol study of cardiovascular disease and oral contraceptives. $\mathcal{F}$ Epidemiol Community Health 1996;50:674-80.

7 World Health Organization Collaborative Study of Cardiovascular Disease and Steroid Hormone Contraception. A vascular Disease and Steroid Hormone Contraception. A
multinational case-control study of cardiovascular disease multinational case-control study of cardiovascular disease dation of methods. F Clin Epidemiol 1995;48:1513-47.
8 Office of Population Censuses and Surveys. Mortality Statisics Area, England and Wales. London: HM Stationary Office, 1989-92 Series DH5 nos 16-19.

9 Thorvaldsen P, Asplund K, Kuulasmaa K, et al, for the WHO MONICA project. Stroke incidence, case fatality, and mortality in the WHO MONICA project. Stroke 1995; 26:361-7.

10 WHO MONICA Project. Myocardial infarction and coronary deaths in the World Health Organization MONICA Project: Registration procedures, event rates and casefatality rates in 38 populations from 21 countries in four continents. Circulation 1994;90:583-612.

11 Stadel BV. Oral contraceptives and cardiovascular disease (part I). N Engl F Med 1981;305:612-18.

12 Sidney S, Petitti DB, Quesenberry CP, et al. Myocardial infarction in users of low-dose oral contraceptives. Obstet Gynecol 1996;88:939-44.

13 Petitti DB, Sidney S, Bernstein A, et al. Stroke in users of low-dose oral contraceptives. N Engl f Med 1996;335:8-15.

14 World Health Organization. World Health Statistics Annual 1992. Geneva: World Health Organization, 1993.

15 World Health Organization. World Health Statistics Annual 1993. Geneva: World Health Organization, 1994

16 Petitti DB, Sidney S, Quesenberry CP, et al. Incidence of stroke and myocardial infarction in women of reproductive age. Stroke 1997;28:280-3

17 Jick H, Jick S, Gurewich V, et al. Risk of idiopathic cardiovascular death and nonfatal venous thromboembolism in women using oral contraceptives with differing progestagen components. Lancet 1995;346:1589-93.

18 Farmer RDT, Lawrenson RA, Thompson RG, et al. Population-based study of risk of venous thromboembolism associated with various oral contraceptives. Lancet 1997;349:83-8.

19 Lidegaard Ø, Milsom I. Oral contraceptives and thrombotic diseases: impact of new epidemiological studies. Contraception 1996;54:195-8.

20 Thorogood M, Mann J, Murphy M, et al. Fatal stroke and use of oral contraceptives: findings from a case-control study. Am $\mathcal{F}$ Epidemiol 1992;136:35-45.

21 Bonita R, Beaglehole R, North JDK. Event, incidence and case fatality rates of cerebrovascular disease in Auckland, New Zealand. Am $\mathcal{F}$ Epidemiol 1984;120:236-43.

22 Schwartz SM, Siscovick DS, Longstreth WT, et al. Use of low-dose oral contraceptives and stroke in young women. Ann Intern Med 1997;127:596-603.

23 Lis Y, Spitzer WO, Mann RD, et al. A concurrent cohort study of oral contraceptive users from the VAMP research bank. Pharmacoepidemiol Drug Safety 1993;2:51-63.

24 Office of Population Censuses and Surveys. Health Survey for England 1993. London: HM Stationary Office, 1993.

25 Bloemenkamp KWM, Rosendaal FR, Helmerhorst FM, et al. Enhancement by factor $\mathrm{V}$ Leiden mutation of risk of deep-vein thrombosis associated with oral contraceptives containing a third-generation progestagen. Lancet 1995; 346:1593-6.

26 Spitzer WO, Lewis MA, Heinemann LA, et al. Third generation oral contraceptives and risk of venous thromboembolic disorders: an international case-control study. Transnational Research Group on Oral Contraceptives and the Health of Young Women. BMF 1996;312:83-8.

27 Thorogood M. Oral contraceptives and cardiovascular disease: an epidemiologic overview. Pharmacoepidemiol Drug Safety 1993;2:3-16.

28 Normand ST, Glickman ME, Sharma RGVRK, et al. Using admission characteristics to predict short-term mortality from myocardial infarction in elderly patients. $¥ A M A$ 1996;275:1322-8

29 Sonke GS, Stewart AW, Beaglehole R, et al. Comparison of case-fatality in smokers and non-smokers after acute case-fatality in smokers and non-
cardiac event. $B M \mathcal{F} 1997 ; 315: 992-3$.

30 Farley TMM, Collins J, Schlesselman JJ. Hormonal contraception and risk of cardiovascular disease: an international perspective. Contraception 1998;57:211-30.

31 Vandenbroucke JP, Koster T, Briët E, et al. Increased risk of enous thrombosis in oral contraceptive users who are carriers of factor V Leiden mutation. Lancet 1994;344:1453-7.

32 Rees DC, Cox M, Clegg JB. World distribution of factor V Leiden. Lancet 1995;346:1133-4.

33 Ridker PM, Miletich JP, Hennekens CH, et al. Ethnic distribution of factor V Leiden in 4047 men and women: Implications for venous thromboembolism screening. $f A M A$ 1997;277:1305-7.

34 Bloemenkamp KWM, Rosendaal FR, Helmerhorst FM, et al. Factor V Leiden and third-generation oral contraceptives. Lancet 1996;347:396-7.

35 Rosing J, Tans G, Nicolaes GAF, et al. Oral contraceptives and venous thrombosis: different sensitivities to activated protein $\mathrm{C}$ in women using second- and third-generation oral contraceptives. Br f Haematol 1997;97:233-8.

36 Lewis MA, Heinemann LAJ, Spitzer WO, et al, for the Transnational Research Group on Oral Contraceptives and the Health of Young Women. The use of oral contraceptives and the occurrence of acute myocardial infarction in young women. Results from the Transnational Study on Oral Contraceptives and the Health of Young Women. Contraception 1997;56:129-40.

37 Collins JA, Gunby J. Oral contraceptive use and the cardiovascular health of Canadian women. I Soc Obstet Gynecol Canada 1997;19:125-37.

38 Schwingl PJ, Shelton J. Modeled estimates of myocardial infarction and venous thromboembolic disease in users of second and third generation oral contraceptives. Contraception 1997;55:125-9. 